\title{
Quasi-exact quantum computation
}

\author{
Dong-Sheng Wang, ${ }^{1}$ Guanyu Zhu $\odot,{ }^{2}$ Cihan Okay, ${ }^{3}$ and Raymond Laflamme ${ }^{1}$ \\ ${ }^{1}$ Institute for Quantum Computing and Department of Physics and Astronomy, University of Waterloo, Waterloo, Ontario N2L 3G1, Canada \\ ${ }^{2}$ IBM T. J. Watson Research Center, Yorktown Heights, New York 10598, USA \\ ${ }^{3}$ Stewart Blusson Quantum Matter Institute and Department of Physics and Astronomy, \\ University of British Columbia, Vancouver, British Columbia V6T 1Z4, Canada
}

(Received 15 October 2019; accepted 2 July 2020; published 22 July 2020)

\begin{abstract}
We study quasi-exact quantum error-correcting codes and quantum computation with them. A quasi-exact code is an approximate code such that it contains a finite number of scaling parameters, the tuning of which can flow it to corresponding exact codes, serving as its fixed points. The computation with a quasi-exact code cannot realize any logical gate to arbitrary accuracy. To overcome this, the notion of quasi-exact universality is proposed, which makes quasi-exact quantum computation a feasible model especially for executing moderatesize algorithms. We find that the incompatibility between universality and transversality of the set of logical gates does not persist in the quasi-exact scenario. A class of covariant quasi-exact codes is defined which proves to support a transversal and quasi-exact universal set of logical gates for $\mathrm{SU}(d)$. This work opens the possibility of quantum computation with quasi-exact universality, transversality, and fault tolerance.
\end{abstract}

DOI: 10.1103/PhysRevResearch.2.033116

\section{INTRODUCTION}

Fault-tolerant quantum computation [1-4] requires quantum codes with error-correction features. One way to introduce fault tolerance is to use transversal gates [5,6]. Unfortunately, it is not possible to find a universal set of logical gates that are transversal [6-8]. Such a "no-go" result is further explored for topological stabilizer codes with local stabilizers $[9,10]$, in which setting transversal gates is extended to gates described by finite-depth local unitary circuits [11-13].

In this work, we explore methods to circumvent the no-go theorems. Methods in the literature mostly focus on relaxing transversality. A common method is to use fault-tolerant measurements, such as magic-state injection [14] and code switching [15]. Another method is to use concatenation [16,17], which achieves fault tolerance without fixed transversality. Quantum computing by non-Abelian anyons [18], realized in lattice systems, implements braidings by finite-depth local circuits [19-21], and hence not transversal.

Transversal gates serve as a primary class of fault-tolerant gates since they do not couple local subsystems, on which independent error correction can be performed, and hence do not spread out local errors. Instead of transversality, in this work we relax the requirements on error correction and universality. As Eastin and Knill originally suggested [6], using approximate error-correcting codes instead of exact codes could be a viable approach to avoid the no-go constraint. In this work, we explore this direction in depth.

Approximate codes [22-34] have been studied in various contexts, and in particular, conditions for approximate

Published by the American Physical Society under the terms of the Creative Commons Attribution 4.0 International license. Further distribution of this work must maintain attribution to the author(s) and the published article's title, journal citation, and DOI. error correction have been studied [25-29]. However, the leftover error after error correction, i.e., recovery error, may not be small. To achieve accurate quantum computation, we have to restrict the setting of approximate codes as follows. We call an approximate code "quasi-exact" if there exists a finite number of tunable scaling parameters such that it can approach exact codes as close as possible. This also implies that the recovery error, which is the accuracy parameter of a code, can be arbitrarily small with welldefined scaling behaviors. Examples of such parameters are system size, local dimension, temperature, and density of states [35].

We find that one has to further relax the definition of universality. Generically, universality means that the group $\mathrm{SU}(d)$ for any $d$ can be realized efficiently to arbitrary accuracy based on a universal gate set. The Solovay-Kitaev algorithm [36] ensures that arbitrary accuracy can be achieved efficiently. We realize that such a definition of universality is strong in the sense that it allows arbitrarily long accurate quantum computation. However, in practice a computation is not arbitrarily long. As a result, we introduce a weak version, coined "quasi-exact" universality, which is suitable for computation of finite length and limited accuracy; however, the accuracy is required to be tunable, hence improving the ability to perform accurate computation of any desired length and eventually approaching universality.

The quasi-exact universality can be supported on quasiexact codes. In the quasi-exact scenario, each logical gate has a finite accuracy and there are in total a finite number of distinct logical gates. There is no infinitesimal logical gate, in particular, which would be treated as a nonperfect logical identity gate. Furthermore, we show that there exist quasiexact codes such that when tuning the scaling parameters, the accuracy parameter becomes smaller and the number of transversal logical gates increases, approaching universality for a certain unitary group. This is in contrast to the exact 
setting for which the size of the transversal logical gate set is fixed.

In this work we find a class of quasi-exact codes which support a set of transversal logical gates for $\mathrm{SU}(d)$. This class of codes are from $\mathrm{SU}(d)$ generalizations of valence-bond solid (VBS) models [37-40], which appear as ground states of one-dimensional (1D) local Hamiltonians with symmetryprotected topological (SPT) orders [41-45] and have proven to be useful in the measurement-based model [46-49]. This class of codes are Lie-group covariant codes, the power (and limitation) of which has been demonstrated recently [30-34]. The SPT order enables covariance and quasi-exact universality, and the local errors we consider can be erasure or arbitrary ones on a local site. The number of logical gates scales as $N d_{q}$, the inverse of the accuracy of the code, for $N$ as the system size and $d_{q}$ as the local dimension. We believe better scaling can be achieved for other types of quasi codes to be found. Our work demonstrates the possibility of fault-tolerant quantum computation with (quasi)universality and transversality.

\section{QUASI CODES}

We first recall the setting of exact codes. An exact encoding can be defined by an isometry $V: \mathcal{H}_{L} \rightarrow \mathcal{H}$ from the logical space $\mathcal{H}_{L}$ to the physical space $\mathcal{H}$ such that $V^{\dagger} V=\mathbb{1}$ and $V V^{\dagger}=P$, for $P$ as the projector onto the code space $\mathcal{C} \subset \mathcal{H}$. The code dimension is denoted as $d_{L}:=\operatorname{dim} \mathcal{H}_{L}=\operatorname{dim} \mathcal{C}$, and the physical dimension is $d_{Q}:=\operatorname{dim} \mathcal{H}$. When $\mathcal{H}=\otimes_{n=1}^{N} \mathcal{H}_{n}$ for a system with $N$ local sites $Q_{n}$ each of dimension $d_{q, n}$, $d_{Q}=\prod_{n} d_{q, n}$.

The correctability condition [50] for a channel $\mathcal{N}$ defined by a set of error operators $\left\{E_{i}\right\}$ on $\mathcal{H}$ is

$$
P E_{i}^{\dagger} E_{j} P=a_{i j} P
$$

for $\left[a_{i j}\right]$ as a density operator. The recovery channel $\mathcal{R}=\left\{R_{k}\right\}$ is found by diagonalizing $\left[a_{i j}\right]$ to be $\left[d_{k}\right]$, and defining

$$
R_{k}:=P F_{k}^{\dagger} / \sqrt{d_{k}}
$$

for $d_{k} \neq 0$ and $\left\{F_{k}\right\}$ as the rotated representation of the noise channel $\mathcal{N}$. Meanwhile, the condition for detection is $P E_{i} P=$ $e_{i} P, e_{i} \in \mathbb{C}$. Each error operator $E_{i}$ could be local or nonlocal. When $\left\{E_{i}\right\}$ is a local basis of the space of bounded operators on a local site $\mathcal{B}\left(\mathcal{H}_{n}\right)$ (the subscript $n$ is omitted for $E_{i}$ ) for any $n$, then for any $F_{j} \in \mathcal{B}\left(\mathcal{H}_{n}\right)$, condition (1) implies $P F_{j}^{\dagger} F_{l} P \propto$ $P$, which means arbitrary local error can be corrected.

Conditions for approximate error correction have been established, and optimizations are needed to find a recovery scheme [24-29], in general. It holds that

$$
P E_{i}^{\dagger} E_{j} P=a_{i j} P+P B_{i j} P
$$

for $\left[a_{i j}\right]$ as a density operator and operators $B_{i j}$ as uncorrectable parts. The exact case (1) is for vanishing $B_{i j}$. The recovery error by a channel $\mathcal{R}$ can be measured by the diamond-norm distance $[51] d_{\diamond}(\mathcal{Q}, \mathbb{1}):=\|\mathcal{Q}-\mathbb{1}\|_{\diamond}$, for $\mathcal{Q}:=\mathcal{V}^{\dagger} \mathcal{R} \mathcal{N} \mathcal{V}, \mathcal{V}(\cdot):=V(\cdot) V^{\dagger}$ as the encoding map, which is equivalent to the trace distance $D_{t}\left(\mathcal{C}_{\mathcal{Q}}, \omega\right)$ by [52]

$$
\frac{1}{2 d_{L}} d_{\diamond}(\mathcal{Q}, \mathbb{1}) \leqslant D_{t}\left(\mathcal{C}_{\mathcal{Q}}, \omega\right) \leqslant \frac{1}{2} d_{\diamond}(\mathcal{Q}, \mathbb{1}),
$$

for $\omega:=|\omega\rangle\langle\omega|,| \omega\rangle:=\sum_{i=1}^{d_{L}}|i i\rangle / \sqrt{d_{L}}$ as the maximally entangled state, and $\mathcal{C}_{\mathcal{Q}}:=\mathcal{Q} \otimes \mathbb{1}(\omega)$, known as a Choi matrix. Other measures can also be employed such as entanglement fidelity $[24,53,54]$ (also see Appendix A). We say an approximate code is $\epsilon$-correctable for a noise channel $\mathcal{N}$ when there exists a recovery channel $\mathcal{R}$ such that $d_{\diamond} \leqslant \epsilon$ [25-29].

Now we introduce quasi-exact codes. We use "quasi" as a shorthand for "quasi-exact." As explained in the Introduction, the motivation is to make the recovery error as small as possible, and the idea is to introduce in some scaling parameters $\vec{\lambda}$ such that the recovery error becomes tunable. The recovery channel $\mathcal{R}$ may depend on $\vec{\lambda}$ in principle; however, in the vicinity of exact codes, we expect that the recovery scheme (2) can be employed. In accordance with the notion of quasi universality to be defined later, we defined quasi codes as follows.

Definition 1: Quasi codes. A quasi code $\mathcal{C}(\vec{\lambda})$ is a family of $\epsilon(\vec{\lambda})$-correctable approximate codes with the recovery scheme (2), such that each code is defined with fixed values of $\vec{\lambda}$, which is a vector of a finite number of real scaling parameters, and $\epsilon(\vec{\lambda}) \rightarrow 0$ at some points in the parameter space of $\vec{\lambda}$. We assume that $\epsilon$ is a smooth function of $\vec{\lambda}$.

We see that the quasi setting is a particular and strengthened case of the approximate setting. We remark on a few points:

(i) We assume the number of scaling parameters is finite.

(ii) A quasi-to-exact (QTE) limit is a limit by tuning some parameters of $\vec{\lambda}$ so that $\epsilon(\vec{\lambda}) \rightarrow 0$ is achieved. Each limiting ("critical") point is an exact code.

(iii) One might demand that $\epsilon$ be a monotonic function of each parameter of $\vec{\lambda}$, while this is not required by definition.

There exists an explicit form of the recovery error in terms of the trace distance $D_{t}\left(\mathcal{C}_{\mathcal{Q}}, \omega\right)$ (4). We find $\mathcal{Q}(\hat{\sigma})=\hat{\sigma}+$ $\sum_{k l} \hat{B}_{k l} \hat{\sigma} \hat{B}_{k l}^{\dagger} / d_{k}$ for any logical state $\hat{\sigma}, \hat{B}_{k l}:=V^{\dagger} B_{k l} V$. This means the logical state is recovered up to the perturbation by the set $\left\{\frac{1}{\sqrt{d_{k}}} \hat{B}_{k l}\right\}$. We obtain the trace distance

$$
D_{t}\left(\mathcal{C}_{\mathcal{Q}}, \omega\right)=\frac{1}{2 d_{L}} \sum_{k l} \frac{1}{d_{k}} \operatorname{tr}\left(\hat{B}_{k l}^{\dagger} \hat{B}_{k l}\right),
$$

which should be a smooth function of each $d_{k}, \beta_{k l}:=$ $\operatorname{tr}\left(\hat{B}_{k l}^{\dagger} \hat{B}_{k l}\right)$, and the cardinality of the set $\{k\}$, i.e., the size of the "environment," $d_{E}$. For local-error models, if the system size is $N$ and local site dimension is $d_{q}$, then $d_{E}=\operatorname{poly}\left(N, d_{q}\right)$; e.g., $N^{c_{1}} d_{q}^{c_{2}}$ to the leading order for some positive constants $c_{1}, c_{2}$. The variables $\beta_{k l}$ and $d_{k}$ should show universal decay behaviors with some scaling parameters such as $N$ and $d_{q}$ in order to make $D_{t}$ small. If $D_{t} \rightarrow 0$, hence $d_{\diamond} \rightarrow 0$, under a QTE limit, it is quasi correctable. This also implies that for bounded operators in the span of $\left\{E_{i}\right\}$, the $D_{t}$ in terms of the new set of $\beta_{k l}^{\prime}$ and $d_{k}^{\prime}$ should also decay in a QTE limit, making it quasi correctable.

\section{QUASI UNIVERSALITY}

Now we introduce the notion of quasi universality, which is suitable for computing with quasi codes, and can also be employed in other settings. Universality means that on a logical space of dimension $d$, the whole group $\mathrm{SU}(d)$ can be 
realized efficiently [55]. The Solovay-Kitaev algorithm [36] proves that, given any $U \in \mathrm{SU}(d)$, there exists an efficient classical algorithm that produces an efficient sequence of logical gates $U^{\prime}=\prod_{i} U_{i}$ with the operator-norm distance $d\left(U, U^{\prime}\right)$ arbitrarily small.

In the setting of universality above, the logical identity gate $\mathbb{1}$ is not explicitly written but implied. However, there is no perfect $\mathbb{1}$ for quasi codes due to the error-correction features [see Eq. (5)]. The leftover from error correction is random, which means that the logical identity gate I should be replaced by a set of gates that are close to it, with a proper distance measure such as entanglement fidelity, trace distance, or operator norm. Denote this set as $\mathfrak{I}_{\eta}$ for $\eta$ as a measure of distance from $\mathbb{1}$. For a quasi code, $\eta$ will be its accuracy $\epsilon(\vec{\lambda})$. The set $\Im_{\eta}$ is treated as an equivalence class of gates, and any gate $\mathbb{1}_{\eta} \in \mathfrak{I}_{\eta}$ is treated the same as $\mathbb{1}$. We call such a set a "gate cell," or just "cell," and $\eta$ the "size" or accuracy of it. Clearly it also induces gate cells for generic logical gates. A gate cell $\mathfrak{U}_{\eta}$ of accuracy $\eta$ for a logical gate $U \in \mathrm{SU}(d)$ is a dense set of gates such that each $U_{\eta} \in \mathfrak{U}_{\eta}$ is of distance $\eta$ from $U$. Therefore, the whole group $\mathrm{SU}(d)$ can be partitioned into a collection of nonoverlapping gate cells of various sizes. One could pick a proper representative gate for each gate cell, and denote the set of them as the coarse-grained set $\mathrm{SU}(d)_{\eta}$, and it is clear that $\mathrm{SU}(d)_{\eta} \subset \mathrm{SU}(d)$. For any $U \in \mathrm{SU}(d)$, there exists an element in $\mathrm{SU}(d)_{\eta}$ of distance no more than $\eta$ from $U$. The partition into cells can be nonuniquely chosen and should be kept fixed in order to define distinct logical gates.

Definition 2: Quasi universality. A computation on a quasi code $\mathcal{C}(\vec{\lambda})$ with accuracy $\epsilon(\vec{\lambda})$ is quasi universal for a unitary group $\mathrm{SU}(d)$ if the coarse-grained set $\mathrm{SU}(d)_{\epsilon(\vec{\lambda})}$ can be realized efficiently, and the group $\mathrm{SU}(d)$ can be approached when $\epsilon(\vec{\lambda}) \rightarrow 0$ in a certain QTE limit.

Generically, for quasi universality the number of distinct logical gates is finite, which yet is quasi infinite due to the ability to improve the accuracy of a quasi code. The transition from quasi universality to universality is not required to be exponentially fast (with respect to some scaling parameters), which is desirable yet not necessary. Different transition behaviors as functions of scaling parameters would lead to a classification of quasi codes that are quasi universal for a certain unitary group.

Arbitrarily long computation cannot be reliably done with quasi codes since the leftover from error correction can accumulate. Let $\eta$ denote the accuracy $\epsilon(\vec{\lambda})$ for simplicity. Suppose we need to approximate $U \in \mathrm{SU}(d)$ to accuracy $\varpi$; what would be the relation between $\varpi$ and $\eta$ ? For quasi codes that any gate $U$ can be realized directly, $\varpi$ is simply $\eta$. For the setting that each gate $U$ is built from a product of gates from a universal gate set, denoted as $\mathcal{S}$, $\varpi$ is lower bounded by $\eta$. Namely, for $U_{\eta}=U \mathbb{1}_{\eta}, V_{\eta}=V \mathbb{1}_{\eta}$, for $\mathbb{1}_{\eta} \in \mathfrak{I}_{\eta}$, the distance $d\left(U_{\eta} V_{\eta}, U V\right) \leqslant 2 \eta$ if $d\left(U_{\eta}, U\right) \leqslant \eta, d\left(V_{\eta}, V\right) \leqslant \eta$ [57]. In general, given any $U \in \mathrm{SU}(d)$, first use gate-synthesis algorithms [36] to approximate $U$ by $U^{\prime}=\prod_{L} \otimes_{J} U_{L J}$, for $U_{L J}$ as gates from a universal gate set $\mathcal{S}, L$ as the label of layers relating to the time of computation, and $J$ as the label of gates in each layer relating to the size of computation. Denote the distance $d\left(U, U^{\prime}\right)$ as $\varpi_{0}$, which could be as small as zero. As each $U_{L J}$ will be approximated by $U_{L J, \eta}$, we see that $d\left(U^{\prime}, U_{\eta}^{\prime}\right) \leqslant m \eta$ for $m:=\sum_{L}|J|_{L}$ as the number of gates. Then the distance $d\left(U, U_{\eta}^{\prime}\right) \leqslant m \eta+\varpi_{0}$. As a result, we could obtain a lower bound for the accuracy

$$
\varpi \geqslant m \eta,
$$

which is also the upper bound on the size $m \leqslant \frac{w}{\eta}$. For the VBS covariant codes that we find below, $\eta$ scales as the inverse of the given system size and local dimension, which provides a linear size constraint on the computation.

\section{TRANSVERSAL LOGICAL GATES}

Now we restrict logical gates to be transversal, which is a primary scheme to achieve fault tolerance. However, it is known that transversality is incompatible with universality [6] (see Appendix B), and this also extends to approximate codes $[33,34]$. With the quasi universality defined above, which is weaker than the requirement of universality, here we argue that transversality is compatible with quasi universality, and there are quasi codes that can achieve both.

We first analyze how quasi error correction modifies the original group-theoretic argument [6]. Transversal gates are of the form

$$
U=\bigotimes_{j} U_{j}
$$

for $j$ as the index of subsystems. The set of transversal logical gates is a Lie group, denoted as $\mathcal{G}$, and the connected component of identity $\mathcal{G}^{0} \subset \mathcal{G}$ is logically trivial for exact codes [6]. We will show now for quasi codes this may not be the case. Let $e^{i \xi D} \in \mathcal{G}^{0}, \xi \in \mathbb{R}$ then $D=\sum_{j} \alpha_{j} H_{j}$ for $H_{j}$ acting on the subsystem $j$, and $D P=P D P$. Given the error set $\left\{E_{j l}\right\}$ on subsystem $j, H_{j}$ can be written as $H_{j}=\sum_{l} \beta_{j l} E_{j l}$. As $P E_{j l} P=e_{j l} P+P B_{j l} P$ from quasi error correction, then

$$
D P=h P+P B P
$$

for $h:=\sum_{j l} \alpha_{j} \beta_{j l} e_{j l}, B:=\sum_{j l} \alpha_{j} \beta_{j l} B_{j l}$. The terms $D^{n} P$ can be computed, and we find $D^{n} P \approx \sum_{m=0}^{n} h^{m} P(B P)^{n-m}$. Thus,

$$
e^{i \xi D} P \approx e^{i \xi h} e^{i \xi P B P} P .
$$

The unitary operator $e^{i \xi P B P}$ is the term due to quasi error correction. If $\xi \in o(1)$, then $e^{i \xi D}$ falls in the cell of logical identity. If $\xi \in O(1)$, then $e^{i \xi D}$ can have a nontrivial logical action. This means that there exist quasi codes for which the connected component of identity $\mathcal{G}^{0} \subset \mathcal{G}$ does not collapse to logical identity. Instead, $\mathcal{G}^{0}$ will permit a gate-cell structure and split into a collection of cells of various sizes. The analysis can also be extended to subsystem quasi codes (Appendix C).

The gate-cell structure of $\mathcal{G}^{0}$ may appear surprising since it seems a tiny recovery error should not affect the logical structure of $\mathcal{G}^{0}$ significantly. The reason is that the recovery error is a measure of the average effects of local noise operators, in terms of a noise channel, while logical gates are from the accumulated net effects of local operators. A logical gate on a quasi code should have a weight (i.e., support) large enough compared with the system size.

As the group $\mathcal{G}$ is compact for a finite system size, the number of logical gates would be finite. This is not a problem for quasi universality since an infinite number of logical gates 
is not required. Furthermore, when a quasi code tends to be exact in a QTE limit, the sizes of gate cells become smaller, and the number of logical operators could tend to be infinite. This is distinct from any exact quantum code for which the number of logical gates is fixed. This demonstrates that there exist quasi codes that can achieve transversality and quasi universality. We see below that the $\mathrm{SU}(d)$-covariant quasi codes achieve this for $\mathrm{SU}(d)$, while the sizes of gate cells for all logical gates are of the same order.

\section{SU(d)-COVARIANT QUASI CODES}

We find classes of covariant codes can be defined from matrix-product states [58,59] with global continuous symmetry, namely, the higher-symmetry generalizations of VBS states [37-40], which have SPT order and appear as ground states of 1D local Hamiltonians. Below we define covariant codes with unitary symmetry, while the construction can be easily generalized to other Lie groups such as orthogonal and symplectic groups, and also to higher spatial dimensions.

The SU $(d)$-covariant codes we consider are defined by the isometry $V:|\alpha\rangle \mapsto\left|\psi_{\alpha}\right\rangle$ for $|\alpha\rangle \in \mathcal{H}_{L}$ and

$$
\left|\psi_{\alpha}\right\rangle=\sum_{i_{1}, \ldots, i_{N}=0}^{d^{2}-1} A_{N}^{i_{N}} \cdots A_{1}^{i_{1}}|\alpha\rangle\left|i_{1}\right\rangle \cdots\left|i_{N}\right\rangle \in \mathcal{C},
$$

with the logical space $\mathcal{H}_{L}$ as the fundamental representation of $\mathrm{SU}(d)$, and the physical space $\mathcal{H}=\mathcal{H}_{L} \otimes\left(\otimes_{n=1}^{N} \mathcal{H}_{n}\right)$, and each $\mathcal{H}_{n}$ as the adjoint representation of the group $\mathrm{SU}(d)$. The $N$ sites $\mathcal{H}_{n}$ are known as "bulk" and the site $\mathcal{H}_{L}$ as "edge." Both the edge and bulk states are employed for the codes. The tensors $A_{n}^{i_{n}}(n=1, \ldots, N)$ are translation invariant (hence $n$ can be omitted), taking the form $A^{i}=\sqrt{\frac{2 d}{d^{2}-1}} t^{i}$ for Gell-Mann matrices $t^{i}$ with $\operatorname{tr}\left(t^{i} t^{j}\right)=\frac{1}{2} \delta_{i j},\left[t^{i}, t^{j}\right]=i f_{i j k} t^{k}$ for $f_{i j k}$ as structure constants of $\mathrm{SU}(d)$. The tensors $A^{i}$, also called Kraus operators, form a quantum channel $\mathcal{E}$ with $\sum_{i} A^{i \dagger} A^{i}=\mathbb{1}$. The symmetry is

$$
\sum_{j} u_{i j}(g) A^{j}=\breve{U}(g) A^{i} \breve{U}(g)^{\dagger}
$$

for $U(g)=\left[u_{i j}\right](g)$ of size $d^{2}-1, \breve{U}(g)$ of size $d$, for $g \in$ $\mathrm{SU}(d)[41,42]$. The channel $\mathcal{E}$ can be dilated to a unitary operator $W$ such that $W|0\rangle=\sum_{i} A^{i}|i\rangle$, which is an isometry. The encoding isometry $V$ is from the product of $W$, each as the dilation of $\mathcal{E}$. From the global symmetry, it is clear that

$$
V \breve{U}(g)|\alpha\rangle=U(g) \otimes U(g) \otimes \cdots \otimes U(g) \otimes \breve{U}(g)\left|\psi_{\alpha}\right\rangle
$$

for $N$ factors of $U(g)$ acting on the bulk and $\breve{U}(g)$ acting on the edge, for $g \in \mathrm{SU}(d)$.

Now we study the error-correction property of the codes. We label the edge as the $(N+1)$ th site, and others from 1 to $N$ sequentially. The edge before encoding can be viewed as the zeroth site. The local state of the edge is

$$
\sigma_{N+1}=\mathcal{E}^{N}(|\alpha\rangle\langle\alpha|)=\mathbb{1} / d+2 \chi^{N} \sum_{a} t^{a} t_{\alpha \alpha}^{a}
$$

for $\chi:=\frac{-1}{d^{2}-1}, t_{\alpha \beta}^{a}:=\left\langle\alpha\left|t^{a}\right| \beta\right\rangle$. This means that partial information of the logical state can be read off from the edge state via $t_{\alpha \alpha}^{a}$, yet this is exponentially suppressed when $N$ increases, and $\sigma_{N+1} \rightarrow \mathbb{1} / d$, which is the unique fixed point of $\mathcal{E}$. The channel $\mathcal{E}$ has other eigenvalues as $\mathcal{E}\left(t^{a}\right)=\chi t^{a}$. The local state $\rho_{n}(n \in[1, N])$ of the $n$th bulk site is

$$
\rho_{n}=\sum_{i j} \operatorname{tr}\left[\sigma_{n} A^{j} A^{i}\right]|i\rangle\langle j|,
$$

which is the complementary state of $\sigma_{n}:=\mathcal{E}^{n-1}(|\alpha\rangle\langle\alpha|)$. It is easy to see $\rho_{n}$ converges to the completely mixed state exponentially, $\rho_{n} \rightarrow \mathbb{1} /\left(d^{2}-1\right)$ as $n$ increases, yet for small $n$, the local state $\rho_{n}$ contains the observable $t_{\alpha \alpha}^{a}$ of the logical state $|\alpha\rangle$. This manifests that the codes are indeed quasi codes.

To define a proper error model, observe that the action of a local operator $T^{a}$, as the adjoint representation of a Gell-Mann matrix $t^{a}$, can be converted to actions on the edge. The action of $T^{a}$ on a local site is $T^{a} \sum_{i} A^{i}|i\rangle=\sum_{i}\left[A^{i}, t^{a}\right]|i\rangle$. Denote the link $(n, n \pm 1)$ as $n \pm$, then the action of $T_{n}^{a}$ on a local site $n$ is a superposition of the actions of $t_{n+}^{a}$ and $t_{n-}^{a}$ on the edge space. So we could view the information encoded in the bulk equivalently as encoded in the links, i.e., the history states of the edge.

For the local error set $\left\{t_{n+}^{a}\right\}$, we find

$$
\left\langle\psi_{\alpha}\left|t_{n+}^{a}\right| \psi_{\beta}\right\rangle=\chi^{n} t_{\alpha \beta}^{a},
$$

and also $\left\langle\psi_{\alpha}\left|t_{n-}^{a}\right| \psi_{\beta}\right\rangle=\chi^{n-1} t_{\alpha \beta}^{a}$. This means local errors are only approximately detected. When $d \rightarrow \infty$, or $n \rightarrow \infty$, the detection becomes exact. For correlation functions we find

$$
\left\langle\psi_{\alpha}\left|t_{m+}^{a} t_{n+}^{b}\right| \psi_{\beta}\right\rangle=\chi^{n-m} \delta_{a b} \delta_{\alpha \beta} / 2 d+\chi^{n} h_{b a c} t_{\alpha \beta}^{c} / 2,
$$

for $n>m \geqslant 0, h_{b a c}=d_{b a c}+i f_{b a c}$, and $d_{b a c}$ are also structure constants of $\mathrm{SU}(d)$. We see that this correlation function contains information $t_{\alpha \beta}^{c}$ of the logical states, which is suppressed for large $n$ or large $d$. In addition, this leads to $\left\langle\psi_{\alpha}\left|T_{n}^{a} t_{N+1}^{b}\right| \psi_{\beta}\right\rangle=\frac{-d \chi^{N-n}}{2\left(d^{2}-1\right)} \delta_{\alpha \beta} \delta_{a b},\left\langle\psi_{\alpha}\left|T_{m}^{a} T_{n}^{b}\right| \psi_{\beta}\right\rangle=$ $\frac{-d^{3} \chi^{n-m-1}}{2\left(d^{2}-1\right)^{2}} \delta_{\alpha \beta} \delta_{a b}$. We see that the edge is more correlated to bulk sites that are close to it, and there is exponential decay of bulk correlation functions.

The quasi conditions (15) and (16) also apply to errors in the span of $\left\{t_{n+}^{a}\right\}$ with $t^{0} \equiv \mathbb{1}$. It is not hard to see that a logical error gate resulting from an error on the link $n+$ depends on $n$, and can be approximated as a unitary operator $E_{n}=e^{i \chi^{n} \sum_{k} \epsilon_{k} t^{k}}, \epsilon_{k} \in \mathbb{R}$. We average over random errors that occur on the system, and the net effect is a random unitary channel

$$
\mathcal{N}(\sigma)=\frac{1}{N} \sum_{n} E_{n} \sigma E_{n}^{\dagger}, \forall \sigma \in \mathcal{B}(\mathcal{C}),
$$

which, to the order $O\left(\chi^{2 n}\right)$ for each $n$, can be approximated by a unitary operator $e^{i \eta \sum_{k} \epsilon_{k} t^{k}}$ for

$$
\eta:=\frac{\chi}{N} \frac{1-\chi^{N}}{1-\chi}
$$

which vanishes when $N \rightarrow \infty$ or $d \rightarrow \infty$ as two types of QTE limits.

The error analysis above is consistent with the erasure error model, for which the performance of covariant codes has been studied [33]. From Eq. (15), we find $\left\langle\psi_{\alpha}\left|T_{n}^{a}\right| \psi_{\beta}\right\rangle=\frac{d^{2} \chi^{n-1}}{d^{2}-1} t_{\alpha \beta}^{a}, \quad\left\langle\psi_{\alpha}\left|t_{N+1}^{a}\right| \psi_{\beta}\right\rangle=\chi^{N} t_{\alpha \beta}^{a}$, and then $t_{\alpha \beta}^{a}=\left\langle\psi_{\alpha}\left|t_{N+1}^{a}\right| \psi_{\beta}\right\rangle+\sum_{n}\left\langle\psi_{\alpha}\left|T_{n}^{a}\right| \psi_{\beta}\right\rangle$. If the environment 
can erase a local site $n \in[1, N+1]$ randomly, then it can read out the logical value $t_{\alpha \beta}^{a}$ from a global observable. Then the uncorrectable part of the code is lower bounded by a quantity proportional to $\left(N \max _{n} \Delta T_{n}\right)^{-1}$, for $\Delta T_{n}$ as the spectral range of a local observable $T_{n}$, and $\max _{n} \Delta T_{n}$ scales with the dimension $d^{2}-1$, which agrees with the scaling of $\eta$ [Eq. (18)].

When the codes are prepared as ground states of frustration-free local Hamiltonians [37-40], which in general take the form $H=\sum_{n} h_{n, n+1}$, the nearest-neighbor interaction terms $h_{n, n+1}$, although they do not commute with each other, play similar roles with stabilizers [60]. Each term $h_{n, n+1}$ is minimized for the code space $\mathcal{C}$. An error $E_{n}$ will increase the energy term $h_{n, n+1}$, and can be corrected by cooling back to $\mathcal{C}$.

For quantum computing with error correction, the sequence of gate operations is $\prod_{\ell=1}^{L} U_{\ell} E_{\ell}$ with the enacted gates $U_{\ell}$ interrupted by error gates $E_{\ell}$. The product of $U_{\ell}$ can yield the whole group $\mathrm{SU}(d)$. Recall that $E_{\ell}$ depends on $\eta$, and when $d \rightarrow \infty$, or $N \rightarrow \infty$, then $E_{\ell} \rightarrow \mathbb{1}$. For finite $d$ and $N$, when the length $L$ of the computation increases, the errors will increase, too. As we already know, the quasi feature limits the accuracy of logical gates. The parameter $\eta$ can be viewed as the unique measure of the accuracy of logical gates, although each error gate $E_{\ell}$ can be different in practice. Therefore, any logical gate is actually a gate cell of size measured by $\eta$, and gates within this accuracy are equivalent. A SU(d)covariant code defined above is quasi universal for $\mathrm{SU}(d)$, and it becomes exact universal for $\mathrm{SU}(d)$ when the code itself approaches exact.

\section{CONCLUSION}

In this work, we have studied the model of quasi-exact quantum computation with two central concepts: quasi codes and quasi universality. The valence-bond solid codes we find can provide a quasicontinuous universal and transversal set of logical gates, with the number of logical gates proportional to the product of the system size and local dimension. We remark that by relaxing the requirement of covariance and allowing various sizes of gate cells, it will naturally lead to the setting that a discrete universal gate set is first realized, and all other gates are from the product of them. It is desirable to find a quasi code that can satisfy the quasi universality, transversality, and discreteness of the gate set simultaneously.

A slightly easier task is to employ concatenation of codes such that their transversal sets of gates can be combined to be universal. The concatenation may also benefit the allowed error threshold. A quasi code can be taken as either an inner code or an outer code, and concatenated with another quasi code or exact code, such as stabilizer codes. It is thus also important to see if there are quasi codes that permit quasi universality with such concatenated transversality.

\section{ACKNOWLEDGMENTS}

This research was supported by the Government of Ontario and the Government of Canada through ISED. We thank an anonymous referee for valuable comments. Discussions with A. M. Alhambra, S. Bravyi, P. Faist, A. Kubica, M. Vasmer, T. Yoder, T. Lan, and Y. Wang are acknowledged.

\section{APPENDIX A: APPROXIMATE QUANTUM ERROR CORRECTION}

According to Ref. [25], a channel $\mathcal{N}$ on a code $P$ is $\epsilon$ correctable if and only if (iff)

$$
P E_{i}^{\dagger} E_{j} P=a_{i j} P+P B_{i j} P
$$

for $\quad d(\mathcal{D}+\mathcal{B}, \mathcal{D}) \leqslant \epsilon, \quad$ with $\quad \mathcal{D}(\rho)=\rho_{*}, \quad \mathcal{B}(\rho)=$ $\sum_{i j} \operatorname{tr}\left(\rho B_{i j}\right)|i\rangle\langle j|, \rho \in \mathcal{C}$, for $\rho_{*}:=\sum_{i j} a_{i j}|i\rangle\langle j|, B_{i j} \in \mathcal{B}(\mathcal{H})$, $d$ as a proper distance measure on channels, e.g., the diamond norm distance $d_{\diamond}$ [51], trace distance on Choi states, or Bures distance $d_{B}=\sqrt{1-F}$ for the entanglement fidelity $F$ [54]. The channels $\mathcal{D}$ and $\mathcal{B}$ map system states to "environment" states since $\{|i\rangle\}$ are states of an environment, $E$. Furthermore, there exists a recovery channel $\mathcal{R}$ such that $d\left(\mathcal{V}^{\dagger} \mathcal{R} \mathcal{N} \mathcal{V}, \mathbb{1}\right) \leqslant \epsilon$. The existence of a near-optimal recovery channel is also proved, but it may require a numerical convex optimization to find it $[24,25]$.

The $\epsilon$-correctability of the set $\left\{E_{i}\right\}$ does not guarantee that for other operators in its span, however. Namely, suppose another set of error operators $\left\{F_{\ell}\right\}$ is defined by a matrix $\Upsilon=\left[y_{\ell i}\right]$ such that $F_{\ell}=\sum_{i} y_{\ell i} E_{i}$. Then the superoperator $\Upsilon$ defined by conjugation of $\Upsilon$ induces two new channels $\tilde{\mathcal{D}}=\Upsilon \circ \mathcal{D}$ and $\tilde{\mathcal{B}}=\Upsilon \circ \mathcal{B}$ to condition (A1) for the new set $\left\{F_{\ell}\right\}$. As $\Upsilon$ is not contractive in general, namely, it does not necessarily reduce distance between states, the distance $d(\tilde{\mathcal{D}}+\tilde{\mathcal{B}}, \tilde{\mathcal{D}})$ is not upper bounded by $\epsilon$ anymore. This poses a problem when considering the approximate correction of arbitrary local errors, and instead motivates the introduction of quasi codes which come with a set of scaling parameters.

\section{APPENDIX B: EASTIN-KNILL NO-GO THEOREM}

We review the contents of the Eastin-Knill no-go theorem [6], which basically states that there is no universal set of transversal logical gates (TLGs) supported by a finitedimensional physical system.

A unitary operator $U$ is a logical operator iff

$$
U P=P U P \text {. }
$$

A unitary operator $U^{\dagger}$ is a logical operator iff $P U=P U P$. It turns out, if $U$ is logical, then $U^{\dagger}$ is also logical. This means a state in the code space cannot be mapped out of it by $U$, while a state out of the code space cannot be mapped into it by $U$ either. It is also equivalent to say $[U, P]=0$.

In order to define transversal gates, we need to introduce a "transversal part," or subsystem. An error-correction subsystem, or subsystem for short, is a part of the whole system for which error correction can be performed. The natural choice of a subsystem is a local site. However, a subsystem can consist of several local sites, which should usually be a connected local part of the whole system. This applies to topological codes that have macroscopic code distances.

A TLG does not couple different nonoverlapping subsystems within the same logical qubit, and only couples a corresponding subsystem from different logical qubits. TLG do not spread out errors across subsystems for the same logical qubit. So the error correction for each logical qubit can ensure fault tolerance. 
For many logical qubits each encoded by a different physical system, denoted as $Q[r]$, usually a one-to-one correspondence of subsystems has to be chosen. For instance, for two logical qubits $Q[r]=\bigcup_{n} Q_{n}[r], r=1,2$, a subsystem $Q_{n}[1]$ can be chosen to correspond to $Q_{n}$ [2] for the same label $n$. In general, there might be a permutation $\pi(n)$. If each system comes with a Hamiltonian $H[r]$, then the total Hamiltonian is the sum of them $H=\sum_{r} H[r]$ without interaction terms.

A transversal gate takes the form

$$
U=\bigotimes_{j} U_{j}
$$

for $j$ as the index of subsystems. Note that $U$ is defined up to any permutation of subsystems since permutation cannot spread out errors, only the locations of them.

In order to see the generality of the Eastin-Knill theorem, below we review its content in details. There are several crucial assumptions:

(a) The error detection is exact for an error set $\left\{E_{i}\right\}$, which spans each local subsystem.

(b) Transversality is fixed; namely, all TLG takes the form (B2) for a given partition of subsystems.

(c) The Hilbert-space dimension of the system is finite.

(d) The universality is exact; i.e., the group $\mathrm{SU}(d)$ is realized efficiently to arbitrary accuracy.

Given a code space $P$, it first shows that the set of logical gates $(\mathrm{B} 1)$ forms a Lie group $\mathcal{L}$. Given a transversality and the form (B2), it shows that the set of TLGs is also a Lie group $\mathcal{G}=\mathcal{L} \cap \mathcal{A}$, for $\mathcal{A}=\bigotimes_{j} U\left(d_{j}\right)$, with $d_{j}$ as the dimension of a subsystem. Now the connected component of identity $\mathcal{G}^{0}$ in $\mathcal{G}$ contains elements of the form $C=\prod_{k} e^{i \xi_{k} D_{k}}, \xi_{k} \in \mathbb{R}$. An operator $e^{i \xi D}$ is a TLG for $\xi \in \mathbb{R}$, and then it shows $D P=$ $P D P$. The operator $D$ can be written as a sum of local terms $D=\sum_{j} \alpha_{j} H_{j}$ due to the structure of Lie algebra, and each $H_{j}$ acts on the subsystem $j$. Given the detection of arbitrary local errors, it holds that $P H_{j} P \propto P$, and then $D P=P D P \propto P$. As the result, $C P \propto P$, which means that $C$ acts as the logical identity gate, and the whole group $\mathcal{G}^{0}$ "collapses" to identity. As the quotient group $\mathcal{Q}=\mathcal{G} / \mathcal{G}^{0}$ is a topologically discrete group, the number of logically distinct operators is finite. In other words, the set of TLGs is not universal.

Next we remark on some points:

(1) During the execution of each logical gate, there may be leakage out of the code space, as long as it goes back to the code space at the end.

(2) Each local unitary $U_{j}$ can be realized in many ways, even not unitarily, as long as the net effect is unitary. For instance, ancilla and measurement can be used.

(3) There is no logical ancilla to realize a logical gate $U$ since $U$ itself must be unitary of the form (B2).

(4) A subsystem can contain several local sites, and this especially applies to codes with large code distance, such as topological codes. Error correction on local sites ensures error correction on a subsystem. For code distance $d=2 t+1$, a subsystem can be as big as $t$. This means that each $U_{j}$ can be an entangling gate on the underlying local sites. However, the transversality has to be fixed to ensure that all logical gates are of the form (B2).

(5) The theorem applies to arbitrarily large but finitedimensional Hilbert space. This fact is crucial to generalize it to the quasi-exact setting; e.g., the group-theoretic argument still applies.

\section{APPENDIX C: SUBSYSTEM QUASI CODES}

Here we extend the results to the subsystem quasi codes, which are the quasi version of subsystem codes. In this case, the code space $\mathcal{C}$ has a tensor product form

$$
\mathcal{C}=\mathcal{T} \otimes \mathcal{J}
$$

for the truly logical subspace $\mathcal{T}$ that encodes the information and a junk "gauge subspace" $\mathcal{J}$ that does not encode logical information. The apparent encoding operator is now not oneto-one, $V:|i\rangle \mapsto\left|\psi_{i}\right\rangle\left|\psi_{j}\right\rangle$, for $|i\rangle \in \mathcal{H}_{L},\left|\psi_{i}\right\rangle \in \mathcal{T}$, and any state $\left|\psi_{j}\right\rangle \in \mathcal{J}$ (which can also be mixed states), but it is effectively one-to-one when the $\mathcal{J}$ part is ignored. The total space takes the form

$$
\mathcal{H} \cong \mathcal{T} \otimes \mathcal{J} \oplus \mathcal{S}
$$

for $\mathcal{S}$ as the syndrome subspace. For error correction, in additional to leakage to $\mathcal{S}$, errors may also generate entanglement between $\mathcal{T}$ and $\mathcal{J}$. A good subsystem code is designed such that $\mathcal{J}$ can benefit the error correction. We use $P, P_{\mathcal{T}}$, and $P_{\mathcal{J}}$ as the projectors on $\mathcal{C}, \mathcal{T}$, and $\mathcal{J}$, respectively. We assume an orthonormal basis of $\mathcal{J}$ can be chosen to define $P_{\mathcal{J}}$.

The exact error correction for a set of errors $\left\{E_{i}\right\}$ on subsystem codes has been shown [61-63] to be

$$
P E_{i}^{\dagger} E_{j} P=\left(\mathbb{1}_{\mathcal{T}} \otimes J_{i j}\right) P,
$$

for $\mathbb{1}_{\mathcal{T}}\left(J_{i j}\right)$ acting on the space $\mathcal{T}(\mathcal{J})$. Contrary to the standard condition (1), here there can be a nontrivial action $J_{i j}$ on the gauge part. The condition above is equivalent to

$$
P_{\mathcal{T}} E_{i g}^{\dagger} E_{j h} P_{\mathcal{T}}=a_{i j g h} P_{\mathcal{T}},
$$

for $E_{i g}:=E_{i}\left|\psi_{g}\right\rangle$, with any $\left|\psi_{g}\right\rangle \in \mathcal{J}$. The effective error operators $E_{i g}$ are rectangular; namely, they can map states in $\mathcal{T}$ to states in $\mathcal{T}$ and $\mathcal{J}$. The span of $\left\{E_{i g}\right\}$ is the product of the span of $\left\{E_{i}\right\}$ and $\left\{\left|\psi_{g}\right\rangle\right\}$. If the dimension of $\mathcal{J}$ is $d_{\mathcal{J}}$, then a set of $d_{\mathcal{J}}^{2}$ linearly independent states $\left\{\left|\psi_{g}\right\rangle\right\}$ is enough to ensure the correction of other errors in the span of $\left\{E_{i g}\right\}$. The recovery channel $\mathcal{R}$ will map states in $\mathcal{T}$ and $\mathcal{J}$ (also $\mathcal{S}$ ) back to states in $\mathcal{T}$. In other words, we see that the modification to the standard condition (1) is to replace square error operators by rectangular ones, and increase the number of them by a factor of $d_{\mathcal{J}}^{2}$.

Unitary logical operators $U$ on subsystem codes not only commute with $P$, but also factorize as

$$
U P=\left(U_{\mathcal{T}} \otimes \mathbb{1}_{\mathcal{J}}\right) P,
$$

for a nontrivial gate $U_{\mathcal{T}}$. The Eastin-Knill theorem holds for subsystem codes, but note that in the proof the projector is $P$ instead of $P_{\mathcal{T}}$, and the actions on the gauge part are ignored. For subsystem quasi codes, there still could be a nontrivial logical gate $e^{i \xi P B P}$ on the code subspace $\mathcal{T}$, in general. 
[1] P. W. Shor, Fault-tolerant quantum computation, in Proceedings of the 37th Conference on Foundations of Computer Science (IEEE, New York, 1996), pp. 56-65.

[2] D. Aharonov and M. Ben-Or, Fault-tolerant quantum computation with constant error, in Proceedings of the 29th Annual ACM Symposium on Theory of Computing (ACM, New York, 1997), pp. 176-188.

[3] E. Knill, R. Laflamme, and W. H. Zurek, Resilient quantum computation: Error models and thresholds, Proc. R. Soc. London A 454, 365 (1998).

[4] J. Preskill, Reliable quantum computers, Proc. R. Soc. London A 454, 385 (1998).

[5] E. Knill, R. Laflamme, and W. Zurek, Threshold accuracy for quantum computation, arXiv:quant-ph/9610011.

[6] B. Eastin and E. Knill, Restrictions on Transversal Encoded Quantum Gate Sets, Phys. Rev. Lett. 102, 110502 (2009).

[7] B. Zeng, A. Cross, and I. L. Chuang, Transversality versus universality for additive quantum codes, IEEE Trans. Inf. Theory 57, 6272 (2011).

[8] X. Chen, H. Chung, A. W. Cross, B. Zeng, and I. L. Chuang, Subsystem stabilizer codes cannot have a universal set of transversal gates for even one encoded qudit, Phys. Rev. A 78, 012353 (2008).

[9] D. Gottesman, Theory of fault-tolerant quantum computation, Phys. Rev. A 57, 127 (1998).

[10] A. Y. Kitaev, Fault-tolerant quantum computation by anyons, Ann. Phys. 303, 2 (2003).

[11] S. Bravyi and R. König, Classification of Topologically Protected Gates for Local Stabilizer Codes, Phys. Rev. Lett. 110, 170503 (2013).

[12] F. Pastawski and B. Yoshida, Fault-tolerant logical gates in quantum error-correcting codes, Phys. Rev. A 91, 012305 (2015).

[13] M. E. Beverland, O. Buerschaper, R. Koenig, F. Pastawski, J. Preskill, and S. Sijher, Protected gates for topological quantum field theories, J. Math. Phys. 57, 022201 (2016).

[14] S. Bravyi and A. Kitaev, Universal quantum computation with ideal Clifford gates and noisy ancillas, Phys. Rev. A 71, 022316 (2005).

[15] A. Paetznick and B. W. Reichardt, Universal Fault-Tolerant Quantum Computation with Only Transversal Gates and Error Correction, Phys. Rev. Lett. 111, 090505 (2013).

[16] E. Knill and R. Laflamme, Concatenated quantum codes, arXiv:quant-ph/9608012.

[17] T. Jochym-O'Connor and R. Laflamme, Using Concatenated Quantum Codes for Universal Fault-Tolerant Quantum Gates, Phys. Rev. Lett. 112, 010505 (2014).

[18] C. Nayak, S. H. Simon, A. Stern, M. Freedman, and S. Das Sarma, Non-Abelian anyons and topological quantum computation, Rev. Mod. Phys. 80, 1083 (2008).

[19] R. Koenig, G. Kuperberg, and B. W. Reichardt, Quantum computation with Turaev-Viro codes, Ann. Phys. 325, 2707 (2010).

[20] N. E. Bonesteel and D. P. DiVincenzo, Quantum circuits for measuring Levin-Wen operators, Phys. Rev. B 86, 165113 (2012).

[21] G. Zhu, M. Hafezi, and M. Barkeshli, Quantum origami: Transversal gates for quantum computation and measurement of topological order, Phys. Rev. Res. 2, 013285 (2020).
[22] D. W. Leung, M. A. Nielsen, I. L. Chuang, and Y. Yamamoto, Approximate quantum error correction can lead to better codes, Phys. Rev. A 56, 2567 (1997).

[23] C. Crépeau, D. Gottesman, and A. Smith, Approximate quantum error-correcting codes and secret sharing schemes, in Annual International Conference on the Theory and Applications of Cryptographic Techniques (Springer, Berlin, 2005), pp. 285301.

[24] M. Reimpell and R. F. Werner, Iterative Optimization of Quantum Error Correcting Codes, Phys. Rev. Lett. 94, 080501 (2005).

[25] C. Bény and O. Oreshkov, General Conditions for Approximate Quantum Error Correction and Near-Optimal Recovery Channels, Phys. Rev. Lett. 104, 120501 (2010).

[26] C. Bény and O. Oreshkov, Approximate simulation of quantum channels, Phys. Rev. A 84, 022333 (2011).

[27] C. Bény, Perturbative Quantum Error Correction, Phys. Rev. Lett. 107, 080501 (2011).

[28] H. Khoon Ng and P. Mandayam, Simple approach to approximate quantum error correction based on the transpose channel, Phys. Rev. A 81, 062342 (2010).

[29] P. Mandayam and H. K. Ng, Towards a unified framework for approximate quantum error correction, Phys. Rev. A 86, 012335 (2012).

[30] J. Preskill, Quantum clock synchronization and quantum error correction, arXiv:quant-ph/0010098.

[31] P. Hayden, S. Nezami, S. Popescu, and G. Salton, Error correction of quantum reference frame information, arXiv: 1709.04471.

[32] F. G. S. L. Brandão, E. Crosson, M. Burak Sahinoğlu, and J. Bowen, Quantum Error Correcting Codes in Eigenstates of Translation-Invariant Spin Chains, Phys. Rev. Lett. 123, 110502 (2019).

[33] P. Faist, S. Nezami, V. V. Albert, G. Salton, F. Pastawski, P. Hayden, and J. Preskill, Continuous symmetries and approximate quantum error correction, arXiv:1902.07714.

[34] M. P. Woods and Á. M. Alhambra, Continuous groups of transversal gates for quantum error correcting codes from finite clock reference frames, Quantum 4, 245 (2020).

[35] We remark that there may not be a sharp distinction between tunable (or controllable) parameters and untunable ones. Such a boundary can depend on technology, for instance.

[36] C. M. Dawson and M. A. Nielsen, The Solovay-Kitaev algorithm, Quantum Inf. Comput. 6, 81 (2006).

[37] I. Affleck, T. Kennedy, E. H. Lieb, and H. Tasaki, Rigorous Results on Valence-Bond Ground States in Antiferromagnets, Phys. Rev. Lett. 59, 799 (1987).

[38] M. Greiter and S. Rachel, Valence bond solids for SU(n) spin chains: Exact models, spinon confinement, and the Haldane gap, Phys. Rev. B 75, 184441 (2007).

[39] H. Katsura, T. Hirano, and V. E. Korepin, Entanglement in an SU(n) valence-bond-solid state, J. Phys. A: Math. Theor. 41, 135304 (2008).

[40] T. Morimoto, H. Ueda, T. Momoi, and A. Furusaki, $\mathbb{Z}_{3}$ symmetry-protected topological phases in the SU(3) AKLT model, Phys. Rev. B 90, 235111 (2014).

[41] X. Chen, Z.-C. Gu, and X.-G. Wen, Classification of gapped symmetric phases in one-dimensional spin systems, Phys. Rev. B 83, 035107 (2011). 
[42] N. Schuch, D. Pérez-García, and I. Cirac, Classifying quantum phases using matrix product states and projected entangled pair states, Phys. Rev. B 84, 165139 (2011).

[43] X. Chen, Z.-C. Gu, Z.-X. Liu, and X.-G. Wen, Symmetry protected topological orders and the group cohomology of their symmetry group, Phys. Rev. B 87, 155114 (2013).

[44] K. Duivenvoorden and T. Quella, Topological phases of spin chains, Phys. Rev. B 87, 125145 (2013).

[45] K. Duivenvoorden and T. Quella, From symmetry-protected topological order to Landau order, Phys. Rev. B 88, 125115 (2013).

[46] R. Raussendorf and H. J. Briegel, A One-Way Quantum Computer, Phys. Rev. Lett. 86, 5188 (2001).

[47] D. Gross and J. Eisert, Novel Schemes for MeasurementBased Quantum Computation, Phys. Rev. Lett. 98, 220503 (2007).

[48] D.-S. Wang, D. T. Stephen, and R. Raussendorf, Qudit quantum computation on matrix product states with global symmetry, Phys. Rev. A 95, 032312 (2017).

[49] D. T. Stephen, D.-S. Wang, A. Prakash, T.-C. Wei, and R. Raussendorf, Computational Power of Symmetry-Protected Topological Phases, Phys. Rev. Lett. 119, 010504 (2017).

[50] E. Knill and R. Laflamme, Theory of quantum error-correcting codes, Phys. Rev. A 55, 900 (1997).

[51] A. Kitaev, A. H. Shen, and M. N. Vyalyi, Classical and Quantum Computation, Graduate Studies in Mathematics Vol. 47 (American Mathematical Society, Providence, RI, 2002).

[52] J. Watrous, The Theory of Quantum Information (Cambridge University Press, Cambrisge, UK, 2018).
[53] A. Gilchrist, N. K. Langford, and M. A. Nielsen, Distance measures to compare real and ideal quantum processes, Phys. Rev. A 71, 062310 (2005).

[54] B. Schumacher, Sending entanglement through noisy quantum channels, Phys. Rev. A 54, 2614 (1996).

[55] Note that there is a subtlety regarding the efficient computability of numbers [56], so that the whole group needs to be replaced by a "punctured" version; here we find we can ignore this without affecting our conclusion.

[56] E. Bernstein and U. Vazirani, Quantum complexity theory, SIAM J. Comput. 26, 1411 (1997).

[57] We assume $\mathbb{1}_{\eta}$ is unitary. If $\mathbb{1}_{\eta}$ is not unitary, we may use distance measures on channels, such as the infidelity and diamond norm, and the results still hold.

[58] D. Perez-Garcia, F. Verstraete, M. M. Wolf, and J. I. Cirac, Matrix product state representations, Quantum Inf. Comput. 7, 401 (2007).

[59] U. Schollwöck, The density-matrix renormalization group in the age of matrix product states, Ann. Phys. 326, 96 (2011).

[60] D.-S. Wang, I. Affleck, and R. Raussendorf, Topological Qubits from Valence Bond Solids, Phys. Rev. Lett. 120, 200503 (2018).

[61] D. Kribs, R. Laflamme, and D. Poulin, Unified and Generalized Approach to Quantum Error Correction, Phys. Rev. Lett. 94, 180501 (2005).

[62] D. Poulin, Stabilizer Formalism for Operator Quantum Error Correction, Phys. Rev. Lett. 95, 230504 (2005).

[63] M. A. Nielsen and D. Poulin, Algebraic and informationtheoretic conditions for operator quantum error correction, Phys. Rev. A 75, 064304 (2007). 